\title{
The absolute continuity of the conjugation of certain diffeomorphisms of the circle
}

\author{
Y. KATZNELSON AND D. ORNSTEIN† \\ Mathematics Department, Stanford University, Stanford CA 94305, USA
}

(Received 22 June 1987 and revised 27 August 1988)

\section{Introduction}

Let $f$ be an orientation preserving $\mathscr{H}^{1}$-diffeomorphism of the circle. If the rotation number $\alpha=\rho(f)$ is irrational and $\log D f$ is of bounded variation then, by a wellknown theorem of Denjoy, $f$ is conjugate to the rigid rotation $R_{\alpha}$. The conjugation means that there exists an essentially unique homeomorphism $h$ of the circle such that $f=h^{-1} R_{\alpha} h$. The general problem of relating the smoothness of $h$ to that of $f$ under suitable diophantine conditions on $\alpha$ has been studied extensively (cf. $\left[\mathrm{H}_{1}\right],[\mathrm{KO}],[\mathbf{Y}]$ and the references given there). At the bottom of the scale of smoothness for $f$ there is a theorem of $\mathrm{M}$. Herman $\left[\mathbf{H}_{\mathbf{2}}\right]$ which states that if $D f$ is absolutely continuous and $D \log D f \in L^{p}, p>1, \alpha=\rho(f)$ is of 'constant type' which means 'the coefficients in the continued fraction expansion of $\alpha$ are bounded', and if $f$ is a perturbation of $R_{\alpha}$, then $h$ is absolutely continuous. Our purpose in this paper is to give a different proof and an improved version of Herman's theorem. The main difference in the result is that we do not need to assume that $f$ is close to $R_{\alpha}$; the proof is very different from Herman's and is very much in the spirit of [KO].

It is not hard to see that the condition of boundedness of the continued-fraction coefficients of $\alpha$ is essential. Given $\alpha$ with unbounded coefficients one can construct $f \in \mathscr{H}^{2}$ such that $h$ is purely singular (see e.g., [HS], [K], [L]).

This paper assumes a general understanding of the dynamics of circle rotations. We shall refer to [KO] for some of the basic facts and notations (but not to the main results of [KO] which assume more smoothness of $f$ and give more for $h$ ).

\section{Notation, terminology and some background}

Our setup is as follows: $f=h^{-1} R_{\alpha} h$ is a diffeomorphism of the circle $\mathbf{T}=\mathbf{R} / \mathbf{Z}, h$ is a homeomorphism and $R_{\alpha}$ is the rigid rotation by $\alpha$. We assume that $\alpha$, which is defined $\bmod 1$, is irrational and, taking a representative in $(0,1)$ we denote by $a_{n}$ the coefficients of the continued fraction expansion of $\alpha$, so that

$$
\alpha=\frac{1}{a_{1}+\frac{1}{a_{2}+\cdots}}
$$

† Research partially supported by NSF Grant No. DMS86-05098. 
and the denominators $q_{n}$ of the convergents satisfyt $q_{n+1}=a_{n} q_{n}+q_{n-1}$.

Definition 1.1. An interval $I=(t, \tau)$ is $q_{n}$-small and its endpoints $t, \tau$ are $q_{n}$-close if $\left\{f^{j}(I)\right\}_{j=0}^{q_{n}-1}$ are disjoint.

One checks easily that $(t, \tau)$ is $q_{n}$-small if, depending on the parity of $n$, either $t \leq \tau \leq f^{q_{n-1}}(t)$ or $f^{q_{n-1}}(\tau) \leq t \leq \tau$. The following simple observation is a convenient starting point for 'the basic procedure' (see e.g. [KO]).

LEMMA 1.1. Let $t, \bar{t} \in \mathbf{T}$ and $n \geq 1$. Then there exist $\tau \in \mathbf{T}$ and an integer $l, 0 \leq l<q_{n}$, such that $\tau$ is $q_{n}$-close to $t$ and $\bar{t}=f^{\prime}(\tau)$.

We assume that $\log D f$ is absolutely continuous and $D \log D f \in L^{p}$ for some $p>1$.

\section{Notations:}

(a) $\mathbf{K}_{n}^{0}=\left\|\log D f^{q_{n}}\right\|_{\infty}$

(b) $\tilde{\tilde{\mathbf{K}}}_{n}^{1}=\operatorname{Sup}\left|\int_{t}^{\tau} D \log D f^{\prime}(s) d s\right|=\sup \left|\log D f^{\prime}(\tau)-\log D f^{\prime}(t)\right| \quad$ the supremum being taken for all $l, 0 \leq l<q_{n}$, and intervals $(t, \tau)$ which are $q_{n}$-small.

(c) $\overline{\mathbf{K}}_{m, n}^{1}=\operatorname{Sup}\left|\int_{t}^{\tau} D \log D f^{\prime}(s) d s\right|$ the supremum is taken now for $l$ of the form $l=c q_{m}<q_{m+1}, m<n$, and intervals $(t, \tau)$ which are $q_{n}$-small.

We have the following

LEMMA 1.2.

$$
\begin{aligned}
& \mathbf{K}_{n}^{0} \leq 2 \tilde{\mathbf{K}}_{n}^{1}, \\
& \tilde{\mathbf{K}}_{n}^{1} \leq \sum_{m=1}^{n-1} \tilde{\overline{\mathbf{K}}}_{m, n}^{1} .
\end{aligned}
$$

Proof. (1.2) follows immediately upon writing an arbitrary $l$ in $\left(0, q_{n}\right)$ as $\sum_{m} c_{m} q_{m}$ with $c_{m} q_{m}<q_{m+1} .(1.1)$ is closely related to Denjoy's original inequality: one uses the fact that for some $\bar{t} \in \mathbf{T}, \log D f^{q_{n}}(\bar{t})=0$, apply lemma 1.1 to obtain $\tau$ and $l$ as described there, write

$$
\log D f^{q_{n}}(t)=\left(\log D f^{q_{n}}(t)-\log D f^{q_{n}}(\tau)\right)+\left(\log D f^{\prime}(\tau)-\log D f^{\prime}\left(f^{q_{n}}(\tau)\right)\right)
$$

and both differences are bounded by $\tilde{\mathbf{K}}_{n}^{1}$.

We denote, for $m<n$,

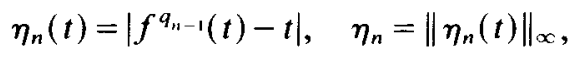

$$
\begin{aligned}
& \eta_{m, n}(t)=\eta_{n}(t) / \eta_{m}(t), \quad \eta_{m, n}=\left\|\eta_{m, n}(t)\right\|_{\infty},
\end{aligned}
$$

and note that (see e.g., [KO] Lemma A.1.1) that there exists $\eta<1$ which depends only on $\operatorname{Var}(\log D f)$ such that for $n-m \geq 2$

$$
\eta_{m, n} \leq \eta^{n-m} \text {. }
$$

LEMMA 1.3. If I is $q_{n}$-small, $m<n$, then, with $\mu=d t$ (the Haar measure of $\mathbf{T}$ ),

$$
\mu\left(\bigcup_{j=0}^{a_{m+1}-1} f^{j}(I)\right) \leq \eta^{n-m} .
$$

\footnotetext{
+ Notice that the coefficient which we denote by $a_{n}$ is denoted by most authors by $a_{n+1}$ (so that, in their notation, $\left.q_{n+1}=a_{n+1} q_{n}+q_{n-1}\right)$.
} 
Proof. Let $I^{\prime}=\left(t_{0}, f^{q_{m}}\left(t_{0}\right)\right)$ be a $q_{m+1}$-interval which contains $I$. By (1.4) the relative length of $I$ in $I^{\prime}$ is $\leq \eta^{n-m}$. The same estimate holds, for the same reason, for the ratio $\mu\left(f^{i}(I)\right) / \mu\left(f^{i}\left(I^{\prime}\right)\right)$ and the lemma follows from the fact that $\sum \mu\left(f^{i}\left(I^{\prime}\right)\right) \leq 1$ since they are disjoint.

Denote $\eta_{1}=\eta^{1-p^{-1}}$.

LEMMA 1.4. $\overline{\mathbf{K}}_{m, n}^{1}=O\left(\eta_{1}^{n-m}\right)$.

Proof. $\overline{\mathbf{K}}_{m, n}^{1}$ is the integral of $D \log D f$ on a set $U=\bigcup_{j=0}^{c_{m, m}^{q_{m}}-1} f^{j}(I)$ and by Lemma $1.3 \mu(U) \leq \eta^{n-m}$. That means, with $p^{-1}+p^{-1}=1$,

$$
\left\|1_{U}\right\|_{p^{\prime}} \leq \eta_{1}^{n-m}
$$

and

$$
\overline{\mathbf{K}}_{m, n}^{1}=\left|\int_{U} D \log D f d t\right|=\left|\int 1_{U} D \log D f d t\right| \leq\left\|1_{U}\right\|_{p^{\prime}}\|D \log D f\|_{p}
$$

which proves the lemma.

In the same way we prove that if $t, \tau$ are $q_{n}$-close, $0 \leq l \leq q_{m}<q_{n}$, then

$$
\left|\log D f^{l}(t)-\log D f^{l}(\tau)\right| \leq \text { const. } \eta_{1}^{n-m} \text {. }
$$

As a corollary to Lemma 1.4 we can replace (1.2) by

$$
\tilde{\mathbf{K}}_{n}^{1} \leq \sum_{m=k}^{n-1} \overline{\overline{\mathbf{K}}}_{m, n}^{1}+O\left(\eta_{1}^{n-k}\right)
$$

2. Condition sufficient for absolute continuity of the conjugation

Definition 2.1. Two measures $\mu, \nu$ on the same $\sigma$-algebra are $L^{2}$-equivalent if $\mu=\varphi_{1} \nu$ with $\varphi_{1} \in L^{2}(\nu)$ and $\nu=\varphi_{2} \mu$ with $\varphi_{2} \in L^{2}(\mu)$.

LeMMA 2.2. $\dagger$ Let $g$ be monotone increasing on $[0,1]$ with $g(0)=0, g(1)=1$. Assume that for some sequence $\left\{b_{n}\right\}$ such that $\sum b_{n}^{2}<\infty$ we have $\ddagger$

$$
\left|\frac{g\left(s+2^{-n}\right)-g(s)}{g(s)-g\left(s-2^{-n}\right)}-1\right|<b_{n} \text { for } 2^{-n} \leq s \leq 1-2^{-n} .
$$

Then $g$ and $\mathrm{g}^{-1}$ are absolutely continuous with square-summable derivatives.

Proof. Denote by $G_{n}$ the linear interpolations of $g$ off $\left\{j 2^{-n}\right\}_{j=0}^{2^{n}}$. Then $\left\{D G_{n}\right\}$ is a martingale (relative to the partitions determined by $\left\{j 2^{-n}\right\}_{j=0}^{2^{n}}, n=1,2, \ldots$ ) and $D G_{n}^{-1}$ is a martingale relative to the $g$-image partitions.

Condition (2.1), for $s=(2 j+1) 2^{-n}$, implies that

$$
\left|\psi_{n}\right|=\left|D G_{n}-D G_{n-1}\right| \leq b_{n} D G_{n-1}
$$

and since $\psi_{n} \perp D G_{n-1}$, we have

$$
\left\|D G_{n}\right\|_{L^{2}}^{2}=\left\|\psi_{n}\right\|_{L^{2}}^{2}+\left\|D G_{n-1}\right\|_{L^{2}}^{2} \leq\left(1+b_{n}^{2}\right)\left\|D G_{n-1}\right\|_{L^{2}}^{2}
$$

and $\left\|D G_{n}\right\|_{L^{2}}^{2} \leq \prod_{1}^{n}\left(1+b_{j}^{2}\right)$. It follows that $D G_{n}$ converges in $L_{2}$ (to $D g$ ). 
The geometric meaning of $\left|\psi_{n}\right| \leq b_{n} D G_{n}$ is that every slope that we see in $G_{n-1}$ is replaced in $G_{n}$ by two slopes, one bigger and one smaller, but the ratios of the new slopes to the preceding lie in $\left(1-b_{n}, 1+b_{n}\right)$. If we look now at the inverse mapping, all the slopes are replaced by their reciprocals and the ratios are now bounded by $\left(\left(1+b_{n}\right)^{-1},\left(1-b_{n}\right)^{-1}\right)$ which is as good as above, and we conclude $D g^{-1} \in L^{2}$, as we did for $D g$.

Remark. The condition $\sum b_{n}^{2}<\infty$ is sharp: given a sequence $\left\{b_{n}\right\}, b_{n}>0$, such that $\sum b_{n}^{2}=\infty$, one can construct a singular $g$ satisfying (2.1) (cf, [C]).

Recall that $h$ denotes the homeomorphism which conjugates $f$ with $R_{\alpha}$, and $d h$ is the $f$-invariant measure on $\mathbf{T}$.

Theorem 2.3. Assume $\sum\left(a_{n} \mathbf{K}_{n}^{0}\right)^{2}<\infty$. Then $d h$ and $d t$ are $L^{2}$-equivalent.

Proof. Without loss of generality we may assume $h(0)=0$ so that $\tilde{h}$, the lifting of $h$ to $\mathbf{R}$, and $\tilde{h}^{-1}$ map $[0,1]$ onto $[0,1]$. We want to apply Lemma 2.2 with $g=\tilde{h}^{-1}$, and we just need to show that the assumption $\sum\left(a_{n} \mathbf{K}_{n}^{0}\right)^{2}<\infty$ implies (2.1) with $\sum b_{n}^{2}<\infty$.

Fix $n$. Take an interval $[t, r]=\left[s-2^{-n}, s+2^{-n}\right]$ and denote its $\tilde{h}$-preimage by $[\tau, \rho]$ and the $\tilde{h}$-preimage of the midpoint $s$ by $\sigma$. We are looking for an estimate $b_{n}$ for $|(\rho-\sigma) /(\sigma-\tau)-1|$, and obtain it through an algorithm to find $\sigma$ using powers of $f$. We use the notation $d_{m}=\left\|q_{m} \alpha\right\|$ (the distance of $q_{m} \alpha$ to the nearest integer on $\mathbf{R}$ or to zero on $\mathbf{T})$ and the relation $a_{m}=\left[d_{m-1} / d_{m}\right]$. Denote by $l$ the smallest integer such that $d_{l}<2^{1-n}=t-r$, and put $c_{l}=\left[2^{1-n} / d_{l}\right]$. Since $2^{1-n} \leq d_{l-1}$, we have $c_{l} \leq a_{l}$. Write $t_{1}=t, r_{1}=r, t_{2}=r_{1}-c_{l} d_{l}, r_{2}=t_{1}+c_{l} d_{l}$, and observe that $\left[t_{1}, t_{2}\right]$ is mapped onto $\left[r_{2}, r_{1}\right]$ by a translation to the right by $c_{l} d_{l}$ which is the same as $R_{ \pm c_{1} q \alpha}$, (the sign depending on the parity of $l)$. Thus $\left(t_{1}, r_{1}\right)$ and $\left(t_{2}, r_{2}\right)$ are concentric and $r_{2}-t_{2}<c_{l} d_{l}$. We now repeat the process for $\left(t_{2}, r_{2}\right)$ : the index $l$ may have increased or remained the same, however, if $l$ remains, that is, $r_{2}-t_{2}>d_{l}$, the parameter $c_{l}$ is certainly lower. Thus we obtain two sequences $\left\{t_{j}\right\}$ and $\left\{r_{j}\right\}$ such that $t_{j+1}>t_{j}$ and $r_{j+1}<r$, and the interval $\left(t_{j}, t_{j+1}\right)$ is mapped onto $\left(r_{j+1}, r_{j}\right)$ by translation to the right by $c_{l, j} d_{l(j)}$, that is, by $R_{ \pm c_{l, j} q_{h(j)} \alpha}$ with $l(j)$ monotone non-decreasing function of $j, c_{l, j} \leq a_{l(j)}$ and is (strictly) decreasing on every $j$-interval on which $l(j)$ is constant. Finally, $r_{j+1}-$ $t_{j+1}<c_{l, j} d_{l(j)}$.

The entire scheme, with $t_{1}=t$ and $r_{1}=r$ is transported by $\tilde{h}^{-1}$ and gives the sequences $\left\{\tau_{i}\right\}$ and $\left\{\rho_{j}\right\}$ the first increasing to $\sigma$, the second decreasing to it, and $\left[\tau_{j}, \tau_{j+1}\right]$ is mapped onto $\left[\rho_{j+1}, \rho_{j}\right]$ by $f^{ \pm c_{l,} q_{(1)}}$. This gives the estimate

$$
\left|\frac{\rho_{j}-\rho_{j+1}}{\tau_{j+1}-\tau_{j}}\right|^{ \pm 1} \leq \exp \left(c_{l, j} \mathbf{K}_{l(j)}^{0}\right) \leq \exp \left(a_{l(j)} \mathbf{K}_{l(j)}^{0}\right)
$$

Combining the estimates (2.2) for all $j$, we obtain

$$
\left|\frac{\rho-\sigma}{\sigma-\tau}-1\right| \leq c_{l} \mathbf{K}_{l}^{0}+\sum_{m=l+1}^{\infty} w_{m} a_{m} \mathbf{K}_{m}^{0}=\bar{b}_{n}+\bar{b}_{n},
$$

where $w_{m}$ are the relative weights of the unions of intervals for which $l(j)=m$. It is not hard to see that $w_{m} \rightarrow 0$ exponentially, in fact since $[\tau, \rho]$ is not $q_{l}$-small 
(though it is $q_{1-1}$-small), and the interval whose relative measure is denoted by $w_{m}$ is $q_{m-1}$-small and is contained in $[\tau, \rho]$, we obtain by (1.4) $w_{m} \leq \eta^{m-1-1}$.

The manipulation of the rest of the proof is simplest in the case of real interest to us, namely when $a_{n}=0(1)$. In this case the parameter $l$, which is defined by $d_{l}<2^{1-n} \leq d_{l-1}$, grows more or less linearly with $n$ (to be precise: any value of $l$ corresponds to at most $L$ values of $n$ or $L$ terms in the martingale, $L$ depends only on the bound for $a_{n}$ ) and the theorem follows from the following (obvious) lemma, putting $\bar{K}_{n}=a_{n} \mathbf{K}_{n}^{0}$.

Lemma 2.4. Assume $\sum_{n=1}^{\infty} \bar{K}_{n}^{2}<\infty$. Define $\bar{b}_{n}=\sum_{l=n}^{\infty} \eta^{1-n} \bar{K}_{l}$, with $0<\eta<1$. Then $\sum \bar{b}_{n}^{2}<\infty$.

The proof in the general case follows from the fact that if an interval $n_{1} \leq n \leq n_{2}$ maintains the same value of $l$, the part $\bar{b}_{n}\left(=c_{l} \mathbf{K}_{l}^{0}\right)$ is largest for $n=n_{1}$ and drops by a factor $\frac{1}{2}$ as we increase $n$ by one. The part $\bar{b}_{n}=\sum_{1+1}^{\infty} w_{m} a_{m} \mathbf{K}_{m}^{0}$ is largest for $n=n_{2}$ and drops by $\frac{1}{2}$ as we decrease $n$ by one. Thus $\sum_{n_{1}}^{n_{2}} \leq 2\left(b_{n_{1}}+b_{n_{2}}\right)$ which brings us back to Lemma 2.4 as before. We leave the details to the reader.

\section{Estimates of $\left\|\log D f^{q_{n}}\right\|_{\infty}$}

Our main goal here is

Theorem 3.1. Assume $D \log D f \in L^{p}$, for some $p>1$. Then

$$
\sum_{n=1}^{\infty}\left(\mathbf{K}_{n}^{0}\right)^{2}<\infty
$$

Notice that we do not assume any diophantine condition on $\alpha$ (except, of course, of being irrational). On the other hand, if the coefficients $a_{n}$ are bounded, then (3.1) implies the condition which, by Theorem 2.3 , guarantees the mutual absolute continuity (in fact the $L^{2}$-equivalence) of $d h$ and $d t$.

We shall make use of the following proposition which seems to be in the spirit of Littlewood-Paley, but as far as we know is new.

Proposition 3.2. Let $\left\{G_{n}\right\}$ be an $L^{p}$-bounded martingale, $1<p \leq 2$. Write $g_{n}=$ $G_{n}-G_{n-1}$. Then

$$
\sum\left\|g_{n}\right\|_{p}^{2}<\infty
$$

We start with

LEMMA 3.3. Let $V$ be a measurable set in a probability space, $g \in L^{p}(V), \int_{v} g d \mu=0$ and $\lambda>0$. Then (the integrals on the right in $V$ ):

$$
\int_{V}\left(|\lambda+g|^{p}-\lambda^{p}\right) d \mu \geq c_{p}\left(\lambda^{p-2} \int_{|g|<\lambda} g^{2} d \mu+\int_{|g| \geq \lambda}|g|^{p} d \mu\right) .
$$

with $c_{p}>0$ depending only on $p$.

Proof. Taylor's theorem with second-order remainder, and direct observation for 
$x<-\lambda$ give

$$
|\lambda+x|^{p}-\lambda^{p}-p \lambda^{p-1} x \geq c_{p} \begin{cases}\lambda^{p-2} x^{2} & \text { for }|x|<\lambda \\ |x|^{p} & \text { for }|x| \geq \lambda\end{cases}
$$

and the lemma follows by writing $g$ for $x$ in (3.4) and integrating over $V$.

Proof of Proposition 3.2. Set $b_{n}=\int\left(\left|G_{n}\right|^{p}-\left|G_{n-1}\right|^{p}\right) d \mu$ so that $\sum b_{n}=\sup \left\|G_{n}\right\|_{p}^{p}$, and apply $\dagger$ Lemma 3.3 to sets $V$ which are level sets for $G_{n-1}$ to obtain

$$
\int_{\left|g_{n}\right|>\left|G_{n-1}\right|}\left|g_{n}\right|^{p} d \mu \leq c_{p}^{-1} b_{n}
$$

and

$$
\int_{\left|g_{n}\right|<\left|G_{n-1}\right|}\left|\frac{g_{n}}{G_{n-1}}\right|^{2}\left|G_{n-1}\right|^{p} d \mu<c_{p}^{-1} b_{n}
$$

and since $\int\left|G_{n-1}\right|^{p} d \mu$ is bounded and $p \leq 2$ the $L^{p}$-norm of $g_{n} / G_{n-1}$ with respect to the measure $\left|G_{n-1}\right|^{p} d \mu$ is bounded by a constant times the $L^{2}$-norm and we obtain

$$
\left(\int_{\left|g_{n}\right|<\left|G_{n-1}\right|}\left|\frac{g_{n}}{G_{n-1}}\right|^{p}\left|G_{n-1}\right|^{p} d \mu\right)^{1 / p} \leq c b_{n}^{1 / 2}
$$

and finally, combining (3.5) and (3.7),

$$
\left(\int\left|g_{n}\right|^{p} d \mu\right)^{2 / p} \leq c b_{n}
$$

which completes the proof.

Proof of Theorem 3.1. By (1.1) it is enough to prove

$$
\sum\left(\tilde{\mathbf{K}}_{n}^{1}\right)^{2}<\infty \text {. }
$$

We propose to prove $\left(3.1^{*}\right)$ by obtaining estimates of $\overline{\mathbf{K}}_{m, n}^{1}$ and then, invoke Lemma 1.2 and (1.8).

So let $n>0$ be arbitrary, $m<n$ (by (1.8) we shall need only consider $n / 2<m<n$ ), $l=c_{m} q_{m}<q_{m+1}$ and $I=(t, \tau)$ which is $q_{n}$-small. By its definition $\overline{\mathbf{K}}_{m, n}$ is the supremum of integrals of the form $\left|\int, D \log D f^{\prime}(s) d s\right|$ and we now fix values of $I$ and $l$ that give the supremum. Keeping in mind that

$$
\int_{I} D \log D f^{\prime}(s) d s=\int_{U} D \log D f(s) d s
$$

with $U=\bigcup_{j=0}^{l-1} f^{j}(I)$, and we can then rewrite $U$ as $\bigcup_{j=0}^{q_{m}-1} f^{j}(E)$ with $E=$

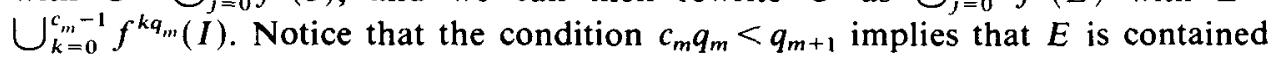
in a $q_{m}$-interval. We now look for a $q_{m}$-interval $J=\left(\bar{t}, f^{q_{m-1}}(\bar{t})\right)$ such that, writing $V=\bigcup_{i=0}^{q_{m}-1} f^{i}(J)$, we have $\int_{V} D \log D f d t=0$, (we obtain it by noting that the integral is equal to $\log D f^{q_{m}}\left(f^{q_{m-1}(}(\bar{t})\right)-\log D f^{q_{m i}}(\bar{t})$ which is continuous in $\bar{t}$, has mean value zero (relative to $d h$ ) and must therefore change signs). The measure $\mu(V)$ is clearly bounded by 1 but, as $V \cup f^{q_{m}}(V)$ is the entire circle and $D f^{q_{m i}}$ is uniformly

$\dagger$ The martingale condition $E\left(g_{n} \mid G_{n-1}\right)=0$ supplies the needed $\int_{V} g d \mu=0$. 
bounded ( $\leq \exp$ (Var $\log D f$ ) by Denjoy's inequality) we obtain also a lower bound and hence by Lemma 1.3 we have

$$
\rho_{m, n}=\mu(U) / \mu(V) \leq C \eta^{n-m},
$$

and writing

$$
\Phi_{m, n}=1_{U}-\rho_{m, n} 1_{V}
$$

we have $\int \Phi_{m, n}(t) d t=0$.

The whole idea of the basic procedure is to evaluate a sum, here taking the form of an integral on a set $U$, by comparing it to one of the same form which is known to vanish. Thus, instead of evaluating $\int 1_{v} D \log D f d s$ we evaluate $\int \Phi_{m, n} D \log D f d s$.

By Lemma 1.1 there exist $\tau$ which is $q_{n}$-close to a point in $I$ and such that $\bar{t}=f^{\prime}(\tau)$ with $0 \leq l<q_{m}$. We write $J^{*}=f^{-l}(J)$ and

$$
j^{*}= \begin{cases}j & l \leq j<q_{m} \\ j+q_{m} & 0 \leq j<l,\end{cases}
$$

so that $V=\bigcup_{j=0}^{q_{m}-1} f^{j^{*}}\left(J^{*}\right)$. The advantage of this notation is that $f^{j^{*}}\left(J^{*}\right)$ is $q_{m}$-close to $f^{j}(E)$ (i.e., some points in the one are $q_{m}$-close to points in the other.)

One can compare $\mu\left(f^{j}(E)\right.$ and $\rho_{m, n} \mu\left(f^{j^{*}}\left(J^{*}\right)\right)$ by noticing first that on the average they are equal, which implies that $\mu\left(f^{j}(E)\right) \geq \rho_{m, n} \mu\left(f^{j^{*}}\left(J^{*}\right)\right)$ for some values of $j$, while the opposite (non-strict) inequality holds for other (values of $j$ ). On the other hand, for any $t_{1} \in f^{j_{1}}(E)$ and $t_{2} \in f^{j_{1}^{*}}\left(J^{*}\right)$, as $t_{1}$ and $t_{2}$ are either $q_{m}$-close or at worst both are $q_{m}$-close to some $t_{3}$; and as for any $j_{2}$ in our range we have $j_{2}^{*}-j_{1}^{*}=j_{2}-j_{1}+$ $\varepsilon q_{m}$ with $\varepsilon= \pm 1$ or zero, we obtain (invoking (1.1) if $\varepsilon \neq 0$ )

$$
\left|\log D f^{j_{2}-j_{1}}\left(t_{1}\right)-\log D f^{j_{2}^{*}-j_{1}^{*}}\left(t_{2}\right)\right|<4 \tilde{\mathbf{K}}_{m}^{1}
$$

which implies

$$
\left|\log \left(\mu\left(f^{j_{2}}(E)\right) / \mu\left(f^{j_{1}}(E)\right)\right)-\log \left(\mu\left(f^{j_{2}^{*}}\left(J^{*}\right)\right) / \mu\left(f^{j_{1}^{*}}\left(J^{*}\right)\right)\right)\right| \leq 4 \tilde{\tilde{\mathbf{K}}}_{m}^{1}
$$

and, since for any $j=j_{2}$ we can find $j_{1}$ such that the signs of

$$
\mu\left(f^{j_{1}}(E)\right)-\rho_{m, n} \mu\left(f^{j_{1}^{*}}\left(J^{*}\right)\right) \quad \text { and } \mu\left(f^{j_{2}}(E)\right)-\rho_{m, n} \mu\left(f^{j_{2}^{*}}\left(J^{*}\right)\right)
$$

are opposite, we obtain

$$
\left|\log \mu\left(f^{j}(E)\right)-\log \left[\rho_{m, n} \mu\left(f^{j^{*}}\left(J^{*}\right)\right)\right]\right| \leq 4 \tilde{\tilde{\mathbf{K}}}_{m}^{1} .
$$

Define $\gamma_{j}, \tilde{\Phi}_{m, n}$ and $\bar{\Phi}_{m, n}$ successively by

$$
\begin{aligned}
\rho_{m, n}\left(1+\gamma_{j}\right) & =\mu\left(f^{j}(E)\right) / \mu\left(f^{j^{*}}\left(J^{*}\right)\right), \\
\tilde{\Phi}_{m, n} & =\rho_{m, n} \sum \gamma_{j} 1_{\left(f^{i^{*}}\left(J^{*}\right)\right)}, \\
\bar{\Phi}_{m, n} & =\Phi_{m, n}+\tilde{\Phi}_{m, n} .
\end{aligned}
$$

Notice that the choice of $\gamma_{j}$ guarantees that

$$
\int \bar{\Phi}_{m, n}=0 \text { on } f^{j}(E) \cup f^{j^{*}}\left(J^{*}\right)
$$

and as $\tilde{\tilde{\mathbf{K}}}_{m}^{1} \rightarrow 0,(3.10)$ implies that (for $m>m_{0}$ )

$$
\left|\gamma_{j}\right| \leq 4 \tilde{\mathbf{K}}_{m}^{1} \text {. }
$$


Remember that we are trying to evaluate $\overline{\mathbf{K}}_{m, n}^{1}$ which is now given as

$$
\overline{\mathbf{K}}_{m, n}^{1}=\left|\int \Phi_{m, n} D \log D f d s\right|=\left|\int\left(\bar{\Phi}_{m, n}-\tilde{\Phi}_{m, n}\right) D \log D f d s\right|
$$

and we estimate separately $\int \bar{\Phi}_{m, n} D \log D f d s$ and $\int \tilde{\Phi}_{m, n} D \log D f d s$. For the latter we need to point out not only that ((3.13))

$$
\left\|\tilde{\Phi}_{m, n}\right\|_{\infty} \leq 4 \rho_{m, n} \tilde{\tilde{K}}_{m}^{1},
$$

but also that $\gamma_{j}$ changes very slowly with $j$. Specifically, if we fix $b$ and impose $\left|j_{1}-j_{2}\right|<q_{b}$ then, by Lemma 1.4 ,

$$
\left|\gamma_{j_{1}}-\gamma_{j_{2}}\right|=O\left(\eta^{m-b}\right)
$$

For sufficiently large $b$ we write $B=\bigcup f^{k q_{h}}(J), k q_{b}<q_{m}$, and by (3.16)

$$
\int \tilde{\Phi}_{m, n} D \log D f d s=\int_{B} \tilde{\Phi}_{m, n} D \log D f^{a_{h}} d s+O\left(\eta^{m-b}\right) \rho_{m, n} .
$$

$B$ is contained in a $q_{b}$-small interval and we can invoke [KO] Theorem 3.9 and (3.15) to obtain for $m>m(\varepsilon)$,

$$
\left|\int \tilde{\Phi}_{m, n} D \log D f d s\right| \leq \varepsilon \rho_{m, n} \tilde{\mathbf{K}}_{m}^{1}+O\left(\eta^{m-b}\right) \rho_{m, n},
$$

where we may take $\varepsilon$ arbitrarily small, (determine $b$ by Theorem 3.9 of [KO] and take $m>b$ ). The only thing we shall want from $\varepsilon$ is to be small enough (less than some constant that we specify later) we can fix it as well as $b$ once and for all, absorb the factor $\eta^{-b}$ into the constant, and remembering that $\rho_{m, n} \leq C_{*} \eta^{n-m}(3.18)$ becomes

$$
\left|\int \tilde{\Phi}_{m, n} D \log D f d s\right| \leq \varepsilon C_{*} \eta^{n-m} \tilde{\tilde{\mathbf{K}}}_{m}^{1}+O\left(\eta^{n}\right) .
$$

For the estimate of $\int \bar{\Phi}_{m, n} D \log D f d s$ we denote by $P_{j}=P_{j}(f)$ the partition of the circle determined by the points $\left\{f^{i}(0)\right\}_{i=0}^{q_{n}-1}$, and by $\left\{G_{j}\right\}$ the martingale expansion of $D \log D f$ relative to $\left\{P_{j}\right\}$ (that means that on each interval-atom of $P_{j}, G_{j}$ is equal to the mean value of $D \log D f$ on that interval). We write $g_{j}=G_{j}-G_{j-1}$ and keep in mind that $g_{j}$ has integral zero on every $P_{j-1}(f)$-interval. As $\left\|G_{j}\right\|_{p} \leq\|D \log D f\|_{p}$ we may apply Proposition 3.2 and conclude that for our specific $\left\{\boldsymbol{g}_{j}\right\},(3.2)$ is valid.

We now estimate $\int \bar{\Phi}_{m, n} g_{j} d s$. Both $\bar{\Phi}_{m, n}$ and $g_{j}$ are simple functions, $g_{j}$ being measurable $P_{j}$ and with integral zero on any $P_{j-1}$ atom. Thus, whenever $\bar{\Phi}_{m, n}$ is constant on a $P_{j-1}$ atom we get no contribution from that atom to $\int \bar{\Phi}_{m, n} g_{j} d s$. Similarly, when $f^{k}(E) \cup f^{k^{*}}\left(J^{*}\right)$ is contained in a $P_{j}$ atom (or, more generally, when $g_{j}$ is constant on $\left.f^{k}(E) \cup f^{k^{*}}\left(J^{*}\right)\right)$ we invoke (3.12) and again get zero contribution to the integral. As we verify below, all this implies:

$$
\left|\int \bar{\Phi}_{m, n} g_{j} d s\right| \leq \begin{cases}C \eta^{n-j}\left\|g_{j}\right\|_{1} & j<m \\ C \eta_{1}^{n-m}\left\|g_{j}\right\|_{p} & n \geq j \geq m . \\ C \eta_{1}^{j-m}\left\|g_{j}\right\|_{p} & j>n\end{cases}
$$

We check this case by case: 
For $j<m$, the contribution to the integral of a given $P_{j}$ atom happen only when $f^{k}(E) \cup f^{k^{*}}\left(J^{*}\right)$ is partly in the atom but not completely, which happens for two values of $k$ at most. $f^{k}(E)$ has relative measure in the atom bounded by $\eta^{n-j}$, $f^{k^{*}}\left(J^{*}\right)$ has its relative measure bounded by $\eta^{m-j}$ and $\Phi_{m, n}$ is bounded by $\rho_{m, n} \leq$ $C \eta^{n-m}$ on it (outside $f^{k}(E)$ ).

For $j \in[m, n]$ the integral on $\bigcup f^{k}(E)$ is estimated as in the proof of Lemma 1.4; that on $\bigcup f^{k^{*}}\left(J^{*}\right)$ is (trivially) much smaller.

For $j>n$ one again estimates the measure of the union of the $P_{j-1}$ atoms on which $\bar{\Phi}_{m, n}$ is not constant.

All that we need to do now is put it all together: by (3.14)

$$
\overline{\mathbf{K}}_{m, n}^{1} \leq\left|\int \bar{\Phi}_{m, n} D \log D f d s\right|+\left|\int \tilde{\Phi}_{m, n} D \log D f d s\right|
$$

and we can estimate the first integral by adding up the estimates (3.20) for all $j$ (recall that $D \log D f=\sum g_{j}$ ), and the second by (3.19) and obtain

$$
\overline{\mathbf{K}}_{m, n}^{1} \leq C\left[\sum_{j<m} \eta^{n-j}\left\|g_{j}\right\|_{p}+\eta_{1}^{n-m} \sum_{j=m}^{n}\left\|g_{j}\right\|_{p}+\sum_{j=n+1}^{\infty} \eta_{1}^{j-m}\left\|g_{j}\right\|_{p}+\varepsilon \eta^{n-m} \tilde{\mathbf{K}}_{m}^{1}+\eta^{n}\right]
$$

with $C$ a constant which depends only on the variation of $\log D f$.

Summing for $m \in[n / 2, n]$ we obtain (see (1.8))

$$
\tilde{\tilde{\mathbf{K}}}_{n}^{1} \leq C \varepsilon \sum_{n / 2<m<n} \eta^{n-m} \tilde{\tilde{\mathbf{K}}}_{m}^{1}+s_{n},
$$

where

$$
s_{n}=\sum_{j} c_{n, j}\left\|g_{j}\right\|_{p}+\frac{n}{2} \eta^{n}
$$

with

$$
c_{n, j} \leq \begin{cases}n \eta^{n / 2} & j<n / 2 \\ \eta_{1}^{n-j} & n / 2 \leq j \leq n \\ \eta_{1}^{j-n} & n \leq j .\end{cases}
$$

By the (trivial) inequality

$$
\left\|\left(b_{n, j}\right)\right\| \leq \sum_{k} \sup _{n}\left|b_{n, n-k}\right|
$$

(the norm of the matrix $\left(b_{n, j}\right)$ is its norm as operator on $\left.l^{2}\right)$ applied to $\left(c_{n, j}\right)$ and by (3.2) we obtain $\sum s_{n}^{2}<\infty$.

By (3.23) the matrix $R$ whose entries are $C \varepsilon \eta^{n-m}$ for $n / 2<m<n$, and zero elsewhere has norm on $l^{2}$ bounded by $2 C \varepsilon(1-\eta)^{-1}<\frac{1}{2}$ for $\varepsilon$ fixed small enough. By (3.22),

$$
(I-R)\left\{\tilde{\tilde{\mathbf{K}}}_{n}^{1}\right\} \in l^{2}
$$

and multiplying by $(I-R)^{-1}$ we obtain $\left\{\tilde{\mathbf{K}}_{n}^{1}\right\} \in l^{2}$. 


\section{REFERENCES}

[C] L. Carleson. On mappings, conformal at the boundary. J. d'Analyse Math. 19 (1967), 1-13.

[ $\left.\mathbf{H}_{1}\right]$ M. R. Herman. Sur la conjugaison différentiable des difféomorphismes du cercle à des rotations. Inst. Hautes Études Sci. Publ. Math. 49 (1979), 5-234.

[ $\left.\mathrm{H}_{2}\right]$ M. R. Herman. Sur les difféomorphismes du cercle de nombre de rotation de type constant. Conference on Harmonic Analysis in Honor of A. Zygmund, Vol. II, (1981), 708-725.

[HS] J. Hawkins \& K. Schmidt. On $C^{2}$-diffeomorphisms which are of type III. Invent. Math. 66 (1982), 511-518.

[K] Y. Katznelson. The action of diffeomorphism of the circle on the Lebesgue measure. J. d'Analyse Math. 36 (1979), 156-166.

[KO] Y. Katznelson \& D. Ornstein. The differentiability of the conjugation of certain diffeomorphisms of the circle. Ergod. Th. \& Dynam. Sys. this issue, preceding paper.

[L] V. F. Lazutkin. Examples of diffeomorphisms of a circle. Vestnik Leningrad Univ. Math. 10 (1982), 55-62.

[Y] J.-C. Yoccoz. Conjugaison différentiable des difféomorphismes du cercle dont le nombre de rotation vérifie une condition diophantienne. Ann. Sci. École Norm. Sup. 417 (1984), 333-359. 